\title{
DEMONSTRATION THAT THE CELL PLASMA RATIO OF BLOOD CONTAINED IN MINUTE VESSELS IS LOWER THAN THAT OF VENOUS BLOOD
}

\author{
By RICHARD V. EBERT AND EUGENE A. STEAD, JR. \\ (From the Medical Clinic of the Peter Bent Brigham Hospital and the Department of Medicine, \\ Harvard Medical School, Boston)
}

(Received for publication December 30, 1940)

A study of the distribution of the red cells in the circulating blood is of both theoretical and practical importance. In spite of the fact that blood taken from artery, vein or finger has the same cell plasma ratio $(1,2)$, certain investigators $(2,3,5)$ believe that the red cells are not uniformly distributed throughout the vascular bed but that the blood from the large vessels has a higher cell plasma ratio than the blood contained in the minute vessels. Direct observation of the minute blood vessels (6) has shown that the red cells flow in the rapidly moving central portion of the stream and that there is a slow moving clear layer of plasma adjacent to the wall of the vessel. Because of the size of this peripheral layer of clear plasma in the minute blood vessels, it has been suggested that the cell plasma ratio of blood contained in the minute vessels is lower than that of blood from the large vessels.

The cell plasma ratio of the blood contained in the minute vessels has not been determined because of the difficulty in obtaining a sample of this blood. Blood collected from a vein or from the cut ends of minute vessels is representative of the blood flowing from the minute vessels but it is not necessarily representative of the blood contained within the minute vessels. The blood contained within the minute vessels consists not only of the central core of red cells and plasma which is flowing rapidly into the veins, but also of the slower moving peripheral layer of plasma.

In this study a technique for obtaining a portion of the blood actually contained within the minute vessels is described. To obtain this blood it is necessary (1) to obstruct the arterial inflow to the part, (2) to empty the blood out of the large vessels, and (3) to force the blood from the minute vessels into the veins where it can be collected. The hemoglobin concentration and protein concentration of the venous blood and of the blood contained within the minute vessels were compared.

\section{METHOD}

Fifteen experiments were performed on six normal male subjects. All determinations were made on fasting subjects who had rested in the horizontal position for $\mathbf{3 0}$ minutes. A blood pressure cuff was placed loosely on the arm, care being taken not to cause venous distention. A needle, with the point toward the wrist, was introduced into the antecubital vein distal to the cuff. In most of the experiments the vein was entered without a tourniquet. When a tourniquet was used, blood was allowed to drip from the needle for 3 minutes before proceeding. A sample of venous blood was then taken. Immediately thereafter the arterial inflow to the arm was stopped by suddenly inflating the cuff on the arm to a pressure of $300 \mathrm{~mm}$. of $\mathrm{Hg}$. The forearm and hand were then elevated and the blood (usually 40 to $60 \mathrm{cc}$.) was removed by a syringe. When no more blood could be obtained, an Esmarch's bandage was applied to the forearm, beginning at the wrist, and the remaining blood was milked toward the needle. About 3 to $6 \mathrm{cc}$. of blood were obtained during the application of the bandage. The protein concentration and hemoglobin concentration of the venous blood and of the blood milked from the forearm were determined. The hematocrit reading of the venous blood and that of the blood squeezed from the forearm were compared in four experiments.

The hemoglobin concentration of the blood was measured by the method of Evelyn (7). With this technique changes in hemoglobin concentration of more than 0.2 gram are significant. The protein was calculated from the specific gravity of the serum by the method of Kagan (8). In three experiments the total nitrogen was determined by the micro-Kjeldahl method, using Nessler's reagent and the Klett photoelectric colorimeter.

\section{RESULTS}

Similar results were obtained in every experiment (Table I). The hemoglobin concentration of blood milked from the minute vessels was from 0.8 to 1.8 grams lower than the hemoglobin concentration of venous blood. In four of the experiments the protein concentration remained unchanged, and in the remainder it decreased slightly. 
TABLE I

The hemoglobin concentration and protein concentration of venous blood and of blood contained within minute vessels

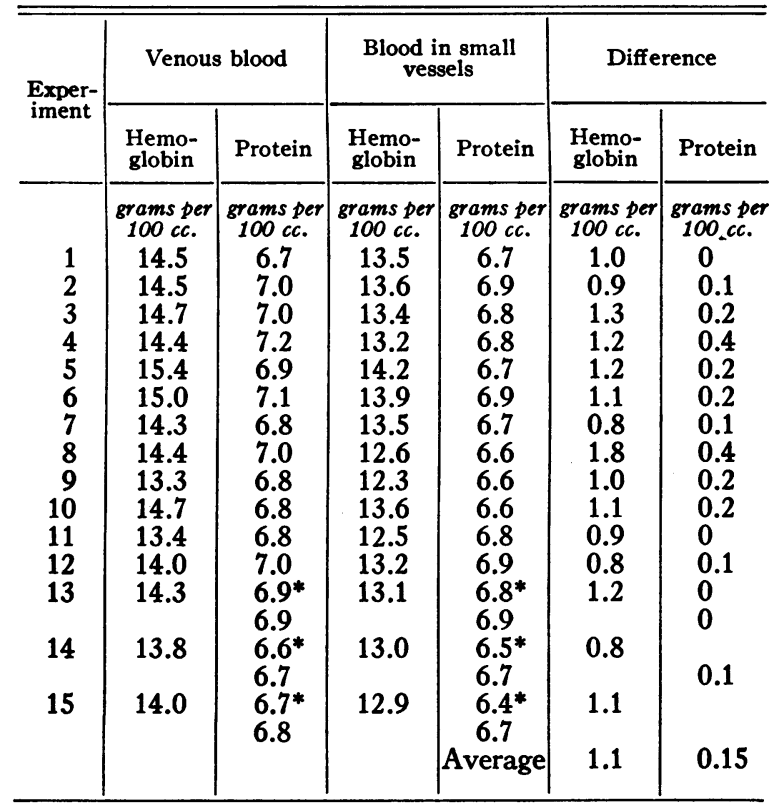

* Done by Kjeldahl method.

This slight fall in protein concentration indicates that fluid was entering the plasma from either the extracellular tissues or from the red cells. As the ratio of the hematocrit reading to the hemoglobin concentration remained the same in the four experiments in which it was determined, the fluid probably came from the extracellular tissues. In no experiment did sufficient fluid enter the plasma to account for more than a small portion of the decrease in hemoglobin concentration. The average decrease in protein would have had to have been approximately six times greater than actually occurred to account for the fall in hemoglobin concentration on the basis of simple dilution with protein-free fluid.

After the arterial inflow to the forearm was occluded, the blood from the forearm was removed with syringes of $10 \mathrm{cc}$. capacity. In three experiments the hemoglobin concentration and protein concentration of the blood contained in each syringe were determined. In each experiment the hemoglobin concentration of the first 20 to $30 \mathrm{cc}$. of blood removed after the application of the tourniquet was the same or slightly less than that of the control sample of venous blood. There- after the hemoglobin concentration showed a progressive fall in successive samples of blood. The protein concentration either remained unchanged or showed an insignificant decrease.

\section{DISCUSSION}

The fact that blood from an artery, vein or finger has, in general, the same cell plasma ratio, has caused many investigators to assume that the hematocrit reading of blood removed from the body is representative of the cell plasma ratio of the entire circulating blood. If this were true, the red cell volume could be calculated from the plasma volume and hematocrit reading. This is not true, for when the red cell volume is calculated from the plasma volume and hematocrit reading, the value obtained for the red cell volume is falsely high $(2,5)$. It has been shown that the error in the calculation of the red cell volume occurs because the hematocrit reading of the blood removed from the body is not representative of the cell plasma ratio of the total quantity of blood in the vascular bed. Smith, Arnold and Whipple (2) compared the value for the red cell volume obtained from the plasma volume and hematocrit reading with that obtained by the carbon monoxide and Welcker methods, and concluded that the value for the red cell volume calculated from the plasma volume and hematocrit reading was approximately 20 per cent higher than the actual red cell volume. Hahn et al. (4) determined the red cell volume of dogs by a method using radio iron. They concluded that the red cell mass in circulation determined by this method averaged about 75 per cent of the value as computed by the jugular hematocrit reading from the plasma volume (dye method). From the plasma volume and hematocrit reading, Stead and Ebert (5) calculated the red cell volume of splenectomized dogs before and after hemorrhage. The pre-hemorrhage red cell volume was from 21 to 34 per cent greater than that obtained from the sum of the post-hemorrhage red cell volume (plasma volume and hematocrit reading) and the volume of red cells removed. The latter determination was believed to approximate the value for the true red cell volume, because 75 per cent of the value for the red cell volume was determined by accurate measurement outside of the 
body, and only 25 per cent was dependent upon the indirect measurement involving the assumption that the hematocrit determines the cell plasma ratio of all the blood in the body. They concluded that with an hematocrit reading of 50 , the value for the red cell volume, as calculated from the plasma volume and hematocrit reading, was approximately 25 per cent higher than the true red cell volume. This study also showed that the value for total circulating hemoglobin obtained from the . total blood volume (plasma volume and hematocrit reading) and hemoglobin concentration of blood removed from the body was approximately 25 per cent higher than the true total circulating hemoglobin. Studies in man have likewise shown that the red cell volume calculated from the plasma volume and hematocrit reading is fasely high (9).

Thus blood volume studies offer indirect evidence that the cell plasma ratio of blood removed from the body does not represent the actual cell plasma ratio of the entire blood. Smith, Arnold and Whipple (2) demonstrated in dogs that this discrepancy is not due to a difference between the hematocrit reading of blood from the extremities and that of blood from the viscera. This must also be true in man because the hematocrit reading of blood from the visceral organs would have to be considerably lower than that in the peripheral blood to account for the discrepancy in red cell volume. If this were true, there would be a sudden fall in the hematocrit reading of blood taken from the extremities when blood is shifted from the viscera by exercise or hemorrhage. This does not occur.

It was suggested by Smith, Arnold and Whipple (2) that the blood flowing in the minute vessels of the body might contain fewer red cells than the blood flowing in the large blood vessels. This idea was supported by the behavior of blood streaming in small vessels. In living tissue under the microscope the red cells are seen to occupy the center of the moving stream and to be surrounded by a clear area of plasma (6). This phenomenon is best seen in vessels of approximately $0.02 \mathrm{~mm}$. in diameter. It can be seen in vessels as large as $0.1 \mathrm{~mm}$. In the minute vessels the clear area of plasma is larger in comparison to total area of the vessel. Fåhraeus (3) showed that, when blood is streaming in small tubes, it is impossible to obtain the true cell plasma ratio of the blood contained in the small tube from the cell plasma ratio of the blood which flows from the cut end of the tube. The axial portion of the stream, which is rich in red cells, flows out of the cut end, while the slower moving peripheral layer of plasma remains in the tube. In his experiments, the hematocrit reading of blood contained in the tube was obtained by sealing the ends of the capillary tube and centrifuging it.

Direct proof that the blood within the minute vessels of the body is poorer in red cells than the blood flowing out of the minute vessels has been lacking. It is possible that there were unknown errors in the methods used to determine the plasma volume and that no thin blood was present in the body. The experiments reported here demonstrate that a portion of the blood usually contained in the minute vessels, in contrast to that normally flowing from these vessels, can be removed from the body and studied. This blood is richer in plasma and poorer in red cells than the blood normally flowing from these vessels. From work on splenectomized dogs, Stead and Ebert (5) concluded that the hemoglobin concentration of blood removed from the body is about 13 per cent higher than the hemoglobin concentration of the entire blood. The hemoglobin concentration of blood in the minute vessels must be considerably lower than that of the entire blood. In the experiments reported here, the hemoglobin concentration of the venous blood was 8 per cent greater than that of the blood from the small vessels. One would not expect the blood obtained by milking the forearm to have as low a cell plasma ratio as actually exists in the small vessels because it is mixed with some blood from the large vessels.

The fact that the cell plasma ratio of the blood in the minute vessels is lower than that of blood in the large vessels makes it impossible to determine accurately the red cell volume, the total circulating hemoglobin, or the total blood volume from the plasma volume, hematocrit reading, and hemoglobin concentration. The calculation of the total blood volume from the plasma volume and hematocrit reading has led to certain erroneous conclusions, particularly in the comparison of the total blood volume of anemic and normal subjects. In pernicious anemia, before liver therapy, the total blood volume, as measured from the plasma volume and hematocrit reading, is on the average 
15 per cent lower than the total blood volume after the hematocrit reading has been restored to normal by the use of liver (10). It appears probable that a large part of this reduction of total volume is apparent rather than real, because after recovery the method gives a falsely high red cell volume, and hence a falsely high total volume. An hematocrit reading, nevertheless, is useful when taken in conjunction with the plasma volume determination because it is known that the plasma volume of otherwise normal individuals varies with the level of the hematocrit reading. It is probable that at rest the distribution of cells and plasma in the large and small vessels is relatively constant. This may account for the observation that the amount of blood removed from the body may be accurately quantitated by determining the plasma volume and hematocrit reading before and after the removal of a known quantity of blood, provided the post-hemorrhage determination is made before the hematocrit reading has fallen appreciably. If the post-hemorrhage determination is made after the hematocrit reading has decreased considerably, it is no longer possible to estimate accurately the amount of blood removed $(9,11)$.

It is not possible to measure accurately changes in plasma volume from changes in hematocrit reading or hemoglobin concentration, as this method is based on the assumption that there is an even distribution of red cells and plasma throughout the body. However, the direction of the change in plasma volume can be determined from the change in the hematocrit reading or hemoglobin concentration. Changes in hematocrit reading or hemoglobin concentration, which are not exactly proportionate to changes in plasma volume or protein concentration, do not mean that red cells have been added or removed from the general circulation. A shift in the proportion of blood present in the large and small vessels can produce changes of this nature. For example, calculation of the red cell volume from the plasma volume and hematocrit reading before and after strenuous exercise shows an apparent increase in the number of red cells in the circulation $(12,13)$. It seems probable that this increase is due to a difference in the distribution of red cells in the circulation because it occurs in splenectomized as well as in normal subjects (13).

\section{SUMMARY AND CONCLUSIONS}

1. A method for obtaining the blood contained in the minute vessels of the forearm is described. A distinction is drawn between blood flowing from the minute vessels and the blood contained within the minute vessels.

2. The venous blood is richer in cells and poorer in plasma than the blood contained within the minute vessels. Therefore, the cell plasma ratio of blood drawn from artery, vein, or finger is not representative of the cell plasma ratio of the entire circulating blood.

3. The value for the red cell volume, as calculated from the plasma volume and hematocrit reading, is falsely high because of the uneven distribution of cells. The value for the total circulating hemoglobin, as calculated on the basis of the plasma volume, hematocrit reading and hemoglobin concentration, is also falsely high.

4. It is not possible to quantitate accurately changes in plasma volume from the changes in hematocrit reading or hemoglobin concentration.

This investigation was carried out with the technical assistance of Miss Rosamond Piotti, S. B.

\section{BIBLIOGRAPHY}

1. Wintrobe, M. M., and Miller, M. W., Normal blood determinations in the South. Arch. Int. Med., 1929, 43, 96.

2. Smith, H. P., Arnold, H. R., and Whipple, G. H., Blood volume studies. VII. Comparative values of Welcker, carbon monoxide and dye methods for blood volume determinations. Accurate estimation of absolute blood volume. Am. J. Physiol., 1921, 56, 336.

3. Fåhraeus, R., The suspension stability of the blood. Physiol. Rev., 1929, 9, 241.

4. Hahn, P. F., Balfour, W. M., Ross, J. F., Bale, W. F., and Whipple, G. H., Red cell volume circulating and total as determined by radio iron. Science, 1941, 93, 87.

5. Stead, E. A., Jr., and Ebert, R. V., Relationship of the plasma volume and the cell plasma ratio to the total red cell volume. Am. J. Physiol., 1941, $132,411$.

6. Jäger, A., Die Anordnung und Stellung der roten Blutkörperchen im strömenden Blut. Arch. f. d. ges. Physiol., 1935, 235, 705.

7. Evelyn, K. A., Stabilized photoelectric colorimeter with light filters. J. Biol. Chem., 1936, 115, 63.

8. Kagan, B. M., Simple method for estimation of total protein content of plasma and serum; estimation of total protein content of human plasma and 
serum by use of falling drop method. J. Clin. Invest., 1938, 17, 373.

9. Ebert, R. V., Stead, E. A., Jr., and Gibson, J. G., 2nd, The response of normal subjects to acute blood loss and the mechanism of the restoration of the blood volume. Arch. Int. Med. (In press.)

10. Gibson, J. G., 2nd, Clinical studies of the blood volume. VI. Changes in blood volume in pernicious anemia in relation to the hematopoietic response to intramuscular liver extract therapy. J. Clin. Invest., 1939, 18, 401.
11. Hooper, C. W., and others, Blood volume studies. I. Experimental control of a dye blood volume method. Am. J. Physiol., 1920, 51, 205.

12. Kaltreider, N. L., and Meneely, G. R., The effect of exercise on the volume of the blood. J. Clin. Invest., 1940, 19, 627.

13. Ebert, R. V., and Stead, E. A., Jr., Demonstration that in normal man no reserves of blood are mobilized by exercise, epinephrine, and hemorrhage. Am. J. M. Sc. (In press.) 